\title{
Probing neutrino magnetic moment at the Jinping neutrino experiment
}

\author{
Baobiao Yue, ${ }^{a}$ Jiajun Liao $^{a, 1}$ and Jiajie Ling ${ }^{a, b, 1}$ \\ ${ }^{a}$ School of Physics, Sun Yat-sen University, \\ No. 135, Xingang Xi Road, Guangzhou, 510275, P.R. China \\ ${ }^{b}$ Key Laboratory of Particle $\&$ Radiation Imaging (Tsinghua University), Ministry of Education, \\ Beijing 100084, China \\ E-mail: yuebb@mail2.sysu.edu.cn, liaojiajun@mail.sysu.edu.cn, \\ lingjj5@mail.sysu.edu.cn
}

ABSTRACT: Neutrino magnetic moment $(\nu \mathrm{MM})$ is an important property of massive neutrinos. The recent anomalous excess at few $\mathrm{keV}$ electronic recoils observed by the XENON1T collaboration might indicate a $\sim 2.2 \times 10^{-11} \mu_{B}$ effective neutrino magnetic moment $\left(\mu_{\nu}^{\text {eff }}\right)$ from solar neutrinos. Therefore, it is essential to carry out the $\nu \mathrm{MM}$ searches at a different experiment to confirm or exclude such a hypothesis. We study the feasibility of doing $\nu \mathrm{MM}$ measurement with 4 kton fiducial mass at Jinping neutrino experiment (Jinping) using electron recoil data from both natural and artificial neutrino sources. The sensitivity of $\mu_{\nu}^{\text {eff }}$ can reach $<1.2 \times 10^{-11} \mu_{B}$ at $90 \%$ C.L. with 10-year data taking of solar neutrinos. Besides the abundance of the intrinsic low energy background ${ }^{14} \mathrm{C}$ and ${ }^{85} \mathrm{Kr}$ in the liquid scintillator, we find the sensitivity to $\nu \mathrm{MM}$ is highly correlated with the systematic uncertainties of $p p$ and ${ }^{85} \mathrm{Kr}$. Reducing systematic uncertainties ( $p p$ and ${ }^{85} \mathrm{Kr}$ ) and the intrinsic background $\left({ }^{14} \mathrm{C}\right.$ and $\left.{ }^{85} \mathrm{Kr}\right)$ can help to improve sensitivities below these levels and reach the region of astrophysical interest. With a 3 mega-Curie (MCi) artificial neutrino source ${ }^{51} \mathrm{Cr}$ installed at Jinping neutrino detector for 55 days, it could give us a sensitivity to the electron neutrino magnetic moment $\left(\mu_{\nu_{e}}\right)$ with $<1.1 \times 10^{-11} \mu_{B}$ at $90 \%$ C.L.. With the combination of those two measurements, the flavor structure of the neutrino magnetic moment can be also probed at Jinping.

Keywords: Neutrino Detectors and Telescopes (experiments)

ArXiv EPrint: 2102.12259

\footnotetext{
${ }^{1}$ Corresponding author.
} 


\section{Contents}

1 Introduction 1

$2 \quad \nu$ MM measurement with solar neutrinos 2

2.1 Jinping neutrino experiment 2

2.2 Solar neutrino signals 3

2.3 Background 5

$\begin{array}{lll}2.4 & \text { Sensitivity } 6\end{array}$

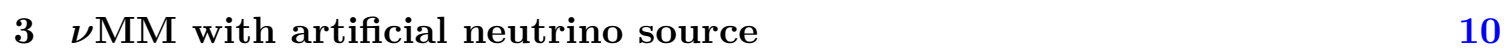

$\begin{array}{ll}3.1{ }^{51} \mathrm{Cr} \text { neutrino signals } & 10\end{array}$

$\begin{array}{lll}3.2 & \text { Sensitivity } & 11\end{array}$

3.3 Combined analyses with 55-day artificial neutrino source and 10-year solar $\begin{array}{ll}\text { neutrinos } & 13\end{array}$

4 Conclusions $\quad 14$

A Electron scattering signal distribution and the effective neutrino $\begin{array}{ll}\text { flux calculation for }{ }^{51} \mathrm{Cr} \text { source } & 15\end{array}$

B An effective neutrino magnetic moment mixing $\mu_{\nu \tau}^{\text {eff }} \quad 16$

\section{Introduction}

Neutrino magnetic moment $(\nu \mathrm{MM})[1-7]$ is closely related to the neutrino mass $m_{\nu}$ [8-13], and neutrino oscillation experiments have verified that the neutrino mass is non-zero, so $\nu \mathrm{MM}$ should theoretically exist. Under the standard electroweak theory, the correlation between $\nu \mathrm{MM}$ and the neutrino mass can be written as

$$
\mu_{\nu}=\frac{3 m_{e} \mathrm{G}_{\mathrm{F}}}{4 \pi^{2} \sqrt{2}} m_{\nu} \mu_{B} \approx 3.2 \times 10^{-19}\left(\frac{m_{\nu}}{\mathrm{eV}}\right) \mu_{B}
$$

where $m_{e}$ is the electron mass, $\mathrm{G}_{\mathrm{F}}$ is the Fermi coupling constant and the Bohr magneton $\mu_{B}$ yields $\mu_{B}=\frac{e \hbar}{4 \pi m_{e}}$. According to eq. (1.1), $\nu \mathrm{MM}$ is less than $10^{-18} \mu_{B}$ order using the upper bound of the current experimental measurement of the neutrino mass [14]. However, some theoretical extensions beyond the Minimally Extended Standard Model (MSM) suggest that if neutrinos are Majorana particles, the magnitude of their magnetic moments would be at the level of $10^{-(10 \sim 12)} \mu_{B}[2,15-18]$. According to general considerations $[19,20]$, the Dirac $\nu \mathrm{MM}$ should have a model-independent and "naturalness" upper limit $\lesssim 10^{-14} \mu_{B}$. Hence if any observation of $\nu \mathrm{MM}$ greater than $10^{-14} \mu_{B}[21,22]$, it would be evidence of new physics and implies that neutrinos might be Majorana particles. 
A previous measurement at Super-Kamiokande reported an upper limit of $\mu_{B}^{\text {eff }}<$ $1.1 \times 10^{-10} \mu_{B}(90 \%$ C.L. $)$ with the SuperK-I solar neutrino data [23]. Gemma experiment bounds electron antineutrino magnetic moment $\left(\bar{\nu}_{e} \mathrm{MM}\right)$ with $<2.9 \times 10^{-11} \mu_{B}(90 \%$ C.L.) using a high purity germanium detecor placed close to a powerful reactor core [24]. Up to now, the Borexino collaboration reported the most stringent upper limit on $\mu_{\nu}^{\text {eff }}$ with $<2.8 \times 10^{-11} \mu_{B}$ (90\% C.L.) [25] among the terrestrial experiments.

Recently, XENON1T observed a $3 \sigma$ event excess [26] at low electron recoil energy, which could be caused by $\nu \mathrm{MM}\left(\mu_{\nu}^{\mathrm{eff}} \in(1.4,2.8)\left[\times 10^{-11} \mu_{B}\right](90 \%\right.$ C.L. $\left.)\right)$ from solar neutrinos. Shortly afterwards, PandaX-II performed a similar study and reported $4.9 \times 10^{-11} \mu_{B}$ (90\% C.L.) [27], which, however, does not reach the sensitive region of $\nu \mathrm{MM}$ reported by XENON1T. On the other hand, astrophysical observations have given tighter limits $<2 \times 10^{-12} \mu_{B}$ (90\% C.L.) [28-31] enough to rule out the abnormal $\nu$ MM in XENON1T. However, the anomaly still needs to be verified by future sensitive terrestrial experiments due to the large uncertainties in astrophysical measurements.

A high-precision measurement of solar neutrinos is proposed by the Jinping neutrino experiment in China, and the accuracy is expected to reach sub-percentage level [32]. In this study we explore the possibility of carrying $\nu \mathrm{MM}$ measurement at Jinping neutrino experiment through solar neutrinos. Besides, we also consider the physics study of deloying a $\mathrm{MCi}$-scale electron-capture neutrino source, ${ }^{51} \mathrm{Cr}[33-38]$, which is a commonly used source in scientific study and can release sub-MeV neutrinos, as proposed by references [39, 40].

The paper is organized as following. Section 2 depicts the study on $\nu \mathrm{MM}$ measurement using solar sources. Section 3 presents the research on $\nu \mathrm{MM}$ with a ${ }^{51} \mathrm{Cr}$ neutrino source. A combined anlysis for solar and artificial neutrino source is also shown in this session. In the end, conclusions are shown in section 4 .

\section{$2 \quad \nu$ MM measurement with solar neutrinos}

\section{$2.1 \quad$ Jinping neutrino experiment}

The Jinping neutrino experiment (Jinping) [32], located in one of the deepest underground laboratories with $2400 \mathrm{~m}$ vertical rock-overburden, aims to study $\mathrm{MeV}$-scale neutrinos, including solar neutrinos, geoneutrinos and supernova neutrinos with the great benefit of ultralow cosmic-ray muon rate. The target material is water-based liquid scintillator (LS), in which Cherenkov light are sensitive to the flight direction of charged particles while scintillation light can be used for the energy and event vertex reconstruction. Ref. [32] assumed three different light yields $(200,500$ and $1000 \mathrm{PE} / \mathrm{MeV})$ in its calculation. In this study, we choose the median, $500 \mathrm{PE} / \mathrm{MeV}$, as the default setup, which corresponds approximately to $4.5 \% / \sqrt{E(\mathrm{MeV})}$ energy resolution. In addition, we also assume the detector has 4 kton fiducial target mass out of 5 kton total mass based on [41]. Compared to Borexino, Jinping is expected to be more sensitive because of a much lower cosmic-ray muon rates and much larger detector mass. 
In terrestrial experiments, $\nu \mathrm{MM}$ is generally detected through the neutrino-electron elastic scattering $(\nu \mathrm{ES})$. The cross section of $\nu \mathrm{ES}$ with $\nu \mathrm{MM}$ can be expressed as

$$
\frac{\mathrm{d} \sigma}{\mathrm{d} T_{e}}\left(T_{e}, E_{\nu}\right)=\underbrace{\frac{\sigma_{0}}{m_{e}}\left[g_{1}^{2}+g_{2}^{2}\left(1-\frac{T_{e}}{E_{\nu}}\right)^{2}-g_{1} g_{2} \frac{m_{e} T_{e}}{E_{\nu}^{2}}\right]}_{\sigma_{\mathrm{SM}}}+\underbrace{\pi \frac{\alpha^{2}}{m_{e}^{2}}\left(\frac{\mu_{\nu}}{\mu_{B}}\right)^{2}\left(\frac{1}{T_{e}}-\frac{1}{E_{\nu}}\right)}_{\sigma_{\nu \mathrm{MM}}} .
$$

In the standard model (SM) cross section term, $T_{e}$ is the kinetic energy of recoil electron, $E_{\nu}$ is the neutrino energy, and $\sigma_{0}$ satisfies $\sigma_{0}=\frac{2 \mathrm{G}_{\mathrm{F}}^{2} m_{e}^{2}}{\pi} \simeq 88.06 \times 10^{-46} \mathrm{~cm}^{2}$. In addition, for $\nu_{e}$ and $\bar{\nu}_{e}, g_{1}$ and $g_{2}$ yields $g_{1}^{\left(\nu_{e}\right)}=g_{2}^{\left(\bar{\nu}_{e}\right)}=\frac{1}{2}+\sin ^{2} \vartheta_{\mathrm{W}} \simeq 0.73$ and $g_{2}^{\left(\nu_{e}\right)}=g_{1}^{\left(\bar{\nu}_{e}\right)}=$ $\sin ^{2} \vartheta_{\mathrm{W}} \simeq 0.23$. Whereas for $\nu_{\mu, \tau}$ and $\bar{\nu}_{\mu, \tau}$, they obey $g_{1}^{\left(\nu_{\mu, \tau}\right)}=g_{2}^{\left(\bar{\nu}_{\mu, \tau}\right)}=-\frac{1}{2}+\sin ^{2} \vartheta_{\mathrm{W}} \simeq$ -0.27 and $g_{2}^{\left(\nu_{\mu, \tau}\right)}=g_{1}^{\left(\bar{\nu}_{\mu, \tau}\right)}=\sin ^{2} \vartheta_{\mathrm{W}} \simeq 0.23$. In $\nu \mathrm{MM}$ cross section term, $\alpha$ is the fine structure constant. More importantly, the $\nu \mathrm{MM}$ cross section from neutrino magnetic moment is proportional to $\left(1 / T_{e}-1 / E_{\nu}\right)$, which indicates lowing detector energy threshold can significantly boost the detection capacity.

\subsection{Solar neutrino signals}

Since Jinping laboratory is far away from nuclear reactor plants, solar neutrinos is the major neutrino source at $\mathrm{MeV}$ energy region. Based on the solar surface metallicity, the standard solar models (SSM) are constructed in two flavors: high metallicity (HZ) and low metallicity (LZ) [42], which predict different solar neutrino fluxes. ${ }^{1}$ In this study, we simply choose the neutrino flux prediction based on HZ hypothesis with $p p\left(5.98(1 \pm 0.006) \times 10^{10}\right)$, ${ }^{7} \mathrm{Be}\left(4.93(1 \pm 0.06) \times 10^{9}\right)$, pep $\left(4.93(1 \pm 0.06) \times 10^{9}\right)$, CNO $\left(4.88(1 \pm 0.11) \times 10^{8}\right),{ }^{8} \mathrm{~B}$ $\left(5.46(1 \pm 0.12) \times 10^{6}\right)$ and hep $\left(7.98(1 \pm 0.30) \times 10^{3}\right)$ in the unit of $\mathrm{cm}^{-2} \mathrm{~s}^{-1}$. In the low energy region, the contributions from $p p$ and ${ }^{7} \mathrm{Be}$ neutrinos dominate the measurement of $\nu \mathrm{MM}$ according to ref. [25]. Other types of solar neutrinos can be ignored.

The signal from the electron and solar neutrino elastic scattering can be calculated with the following formula

$$
N_{\mathrm{pre}}\left(T_{e}\right)=N_{e} T \sum_{i} \phi_{i} \int S_{i}^{\odot}\left(E_{\nu}\right) \sum_{\alpha=e, \mu, \tau} P_{e \alpha}^{i}\left(E_{\nu}\right) \sigma_{\alpha}\left(E_{\nu}, T_{e}\right) d E
$$

where $N_{e}$ is the total electron number of the fiducial volume, yielding $N_{e}=V \rho_{\mathrm{LS}} \rho_{e} \mathrm{~N}_{\mathrm{A}}=$ $1.35 \times 10^{33}$ with the total volume $V$, the exposure time $T$, the LS density $\rho_{\mathrm{LS}}$, the electron density $\rho_{e}(\mathrm{~mol} / \mathrm{g})$ and the Avogadro constant $\mathrm{N}_{\mathrm{A}}$. $i$ is the $i^{\text {th }}$ solar neutrino source. $\phi_{i}$ is the corresponding neutrino flux. $S_{i}^{\odot}$ is the normalized energy spectrum of each solar neutrino. $P_{e \alpha}^{i}\left(E_{\nu}\right)$ is the oscillation probability of the $i^{\text {th }}$ solar neutrinos propagating from the Sun to the earth, which has taken into account the distributions of energy spectrum and birth location in the sun. The oscillation probability can be expressed as

$$
P_{e \alpha}^{i}\left(E_{\nu}\right)=\int_{0}^{R_{\odot}} F_{i}(r) P_{e \alpha}^{\odot}\left(r, E_{\nu}\right) d r
$$

\footnotetext{
${ }^{1}$ We checked that the choice of metallicity has negligible effect on the sensitivity of $\nu \mathrm{MM}$ detection.
} 


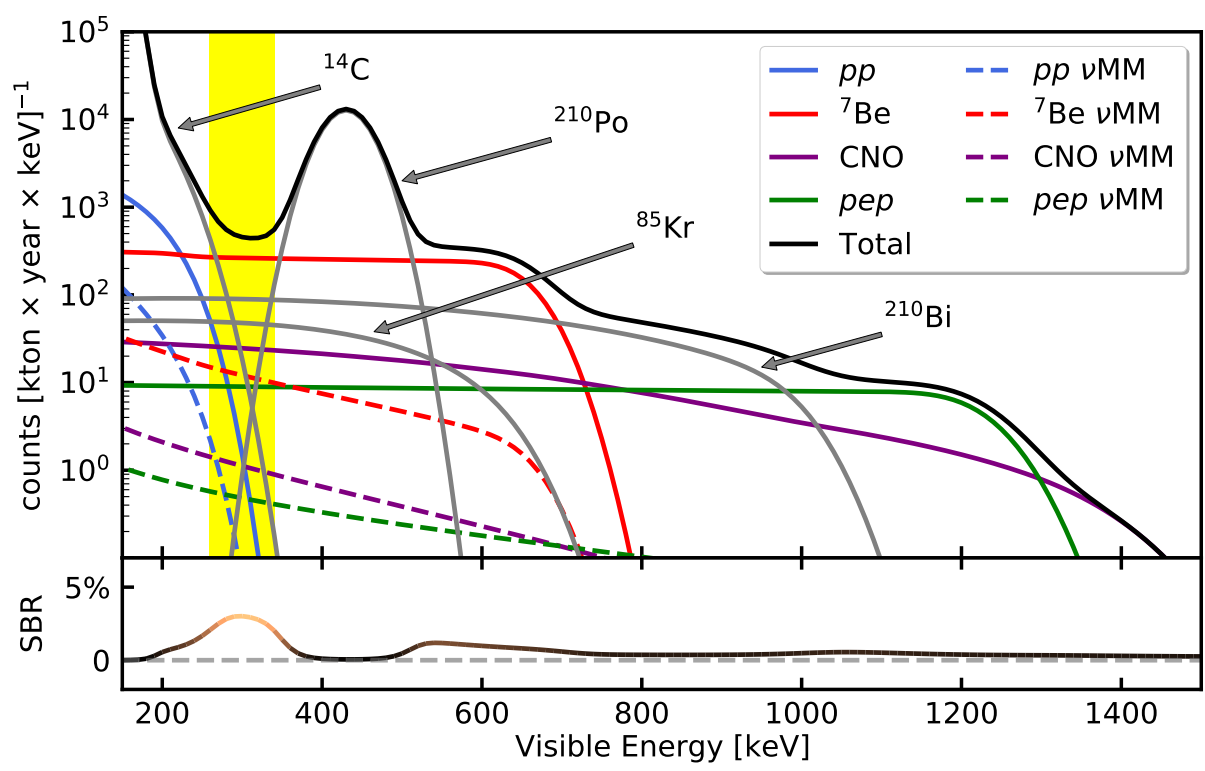

Figure 1. Energy distribution from various solar neutrino components and backgrounds. $4.5 \% / \sqrt{E(\mathrm{MeV})}$ energy resolution has been considered. The dashed lines correspond to the contributions of the magnetic moment from their sources with the same color. The target signal is the electronic recoil caused by $\nu \mathrm{MM}$ from all solar neutrinos. The SM ES signal is also background for $\nu \mathrm{MM}$. The yellow band represents the region with the relative large $\nu \mathrm{MM}$ signal-to-background ratio shown in the subplot for all neutrinos, assuming $\mu_{\nu}^{\text {eff }}=2.2 \times 10^{-11} \mu_{B}$, which is the best fit of $\nu \mathrm{MM}$ hypothesis in XENON1T [26].

where $R_{\odot}$ is the radius of the Sun, $F_{i}(r)$ is the weight of $i^{\text {th }}$ neutrino distribution [43] as a function of radius $r$ in the Sun, and $P_{e \alpha}^{\odot}\left(r, E_{\nu}\right)$ is the oscillation probability of a neutrino produced at $r$ with energy $E_{\nu}$ and detected at the Earth. Since the energy of $p p$ and ${ }^{7} \mathrm{Be}$ neutrino is very low $\left(E_{\nu}^{\max }<1 \mathrm{MeV}\right)$, the matter effects from the Earth can be ignored. Therefore, $P_{e \alpha}^{\odot}\left(r, E_{\nu}\right)$ takes no account of the day-night effect. $\sigma_{\alpha}$ is the cross section of $\nu \mathrm{ES}$ for $\alpha$-flavor neutrinos.

In this study, we assume $100 \%$ detection efficiency in the fiducial volume. Figure 1 shows the energy spectrum for each solar neutrino component and also for backgrounds. The input oscillation parameters are taken from PDG 2020 [44]. In order to measure $\nu \mathrm{MM}$ precisely, it is crucial to evaluate accurately all components between 250 and $300 \mathrm{keV}$, which is the most sensitive region of interest (ROI) as shown with the yellow band in figure 1 . Given the fact that the energy spectrum of $\mathrm{SM} \nu \mathrm{ES}$ from $p p$ is similar to the one from $\nu \mathrm{MM}$ ES signal, an external constraint on the $p p$ neutrino flux can play an important role in the detection of $\nu \mathrm{MM}$. The flux of $p p$ neutrino can be mainly limited by the radiochemical constraints with $5 \%$ from gallium experiments [45]. The SM electron recoil of the ${ }^{7} \mathrm{Be}$ neutrino gives a clear "shoulder" structure in the energy region of 500 to $700 \mathrm{keV}$, which helps to determine the flux of the ${ }^{7} \mathrm{Be}$ neutrino accurately. The fluxes of CNO and pep could be measured from 900 to $1300 \mathrm{keV}$ region, where the signal contribution from $\nu \mathrm{MM}$ is negligible. 


\begin{tabular}{|c|c|}
\hline Background & Rate [cpd/kton] \\
\hline${ }^{14} \mathbf{C}$ & $3.456 \times 10^{7}$ \\
${ }^{85} \mathbf{K r}$ & 68 \\
${ }^{210} \mathbf{B i}$ & 175 \\
${ }^{210} \mathbf{P o}$ & 2600 \\
\hline
\end{tabular}

Table 1. Background rates taken from Borexino Phase-II [46].

\subsection{Background}

In general, three types of background should be considered: cosmogenic background induced by cosmic-ray muons, internal and external radioactive backgrounds. In addition, for the study of $\nu \mathrm{MM}$, the SM $\nu \mathrm{ES}$ process becomes the intrinsic background to $\nu \mathrm{MM}$. Cosmogenic background has small impact on the $\nu \mathrm{MM}$ measurement. The cosmogenic background induced by cosmic-ray muons is about 200 times lower than that in Borexino [32]. We assume the internal radioactive background can reach the same level as Borexino Phase-II [46] after purification. The intrinsic ${ }^{14} \mathrm{C}$ of LS is $2.7 \times 10^{-18} \mathrm{~g} / \mathrm{g}$ in Borexino. The external $\gamma$-rays can be predicted with an exponential scaling factor according to the distance from the edge of the fiducial volume to the detector surface. Because of the self-shielding effect from the large detector size of Jinping, we can safely ignore this background for this analysis. For $\nu \mathrm{MM}$ research, the internal ${ }^{14} \mathrm{C},{ }^{210} \mathrm{Bi},{ }^{85} \mathrm{Kr}$ and ${ }^{210} \mathrm{Po}$ radioactivities are the major backgrounds as shown in table 1. For the low energy study, we also consider the pile-up effect from ${ }^{14} \mathrm{C}-{ }^{14} \mathrm{C}$. With $200 \mathrm{~ns}$ charge integration window $(\delta t)$ assumed, we can roughly estimate the rate of the ${ }^{14} \mathrm{C}-{ }^{14} \mathrm{C}$ pile-up as

$$
R_{\text {pile-up }} \approx 2 \times \frac{\left(R_{{ }_{14} \mathrm{C}} \times M_{F V}\right) \times\left(R_{{ }_{14} \mathrm{C}} \times M_{T V}\right) \times \delta t}{M_{F V}} \approx 28000 \mathrm{cpd} / \text { kton },
$$

where $M_{F V}$ is the target mass in the fiducial volume and $M_{T V}$ is the total target mass of full volume. The energy spectrum is generated with self-convolution of the ${ }^{14} \mathrm{C}$ spectrum. According ref. [47], other pile-up events, mainly ${ }^{14} \mathrm{C}$ and ${ }^{210} \mathrm{Po}$ piling up with external $\gamma$-rays, are negligible.

The rate of ${ }^{14} \mathrm{C}$ could be measured independently as Borexino [48]. Due to large abundance of ${ }^{14} \mathrm{C}$ below $150 \mathrm{keV}$, signal detection at such low energy region is infeasible. Thanks to the good enegy resolution at Jinping, ${ }^{14} \mathrm{C}$ itself does not severely leaked above $150 \mathrm{keV}$ to affect $p p$ and ${ }^{7} \mathrm{Be}$ neutrinos. However, the measurement of $p p$ remains a challenge due to the pile-up of ${ }^{14} \mathrm{C}-{ }^{14} \mathrm{C}$. For the ideal study, we temporarily ignore the shape uncertainty of ${ }^{14} \mathrm{C}$ [49]. ${ }^{210} \mathrm{Po}$ is the dominant background in the $350-550 \mathrm{keV}$ range, which can be clearly fitted with a gaussian distribution. In addition, the $\alpha$ particle from ${ }^{210} \mathrm{Po}$ could be discriminated with scintillation pulse shape from $e^{ \pm}$. To be conservative, we still consider ${ }^{210} \mathrm{Po}$ as a background in this study. Because of the signature of "shoulder" structure of ${ }^{210} \mathrm{Bi}$ in the region from 700 to $1000 \mathrm{keV}$ and its large quantity, they can be properly measured through spectrum fitting. 
As we mentioned above, the region between ${ }^{14} \mathrm{C}$ and ${ }^{210} \mathrm{Po}$ is selected as the ROI of $\nu \mathrm{MM}$ measurement. However, ${ }^{85} \mathrm{Kr}$ is hidden beneath all the other components of the ROI, making it difficult to measure. In addition, It is almost free to mimic the shape of $\nu \mathrm{MM}$ component from ${ }^{7} \mathrm{Be}$ in the ROI. That is to say, the residual ${ }^{85} \mathrm{Kr}$ can significantly affect the $\nu \mathrm{MM}$ measurement. Therefore, good liquid scintillator purification and independent measurement of ${ }^{85} \mathrm{Kr}$ can accordingly improve the capability of $\nu \mathrm{MM}$ measurement. However, purification of ${ }^{85} \mathrm{Kr}$ is generally difficult. $0.4 \%$ decay rate of ${ }^{85} \mathrm{Kr}-{ }^{85} \mathrm{~m}$ Rb delayed coincidence signal can be used to measure ${ }^{85} \mathrm{Kr}$ quantity in-situ. Borexino [46] calculated that it can be picked out with an efficiency of $18 \%$, which allows 10-year independent measurement to put a $4 \%$ constraint on the rate of ${ }^{85} \mathrm{Kr}$.

\subsection{Sensitivity}

For the sensitivity study, we build a $\chi^{2}$ function as

$$
\chi^{2}=\sum_{i}^{T_{e}} \frac{\left(N_{\mathrm{pre}}^{i}-N_{\mathrm{obs}}^{i}\right)^{2}}{N_{\mathrm{obs}}^{i}}+\sum_{\alpha}\left(\frac{\delta_{\alpha}}{\sigma_{\alpha}}\right)^{2},
$$

where $N_{\text {pre }}^{i}$ and $N_{\text {obs }}^{i}$ are the event numbers in the $i^{\text {th }}$ bin of the prediction and the observation with visible energy from 150 to $1500 \mathrm{keV}$, and $\left(\frac{\delta_{\alpha}}{\sigma_{\alpha}}\right)^{2}$ is the penalty term to constrain systematic terms, such as solar neutrino oscillation parameters and solar neutrino flux. In which, $\delta_{\alpha}$ represents the deviation of parameter $\alpha$ away from its prior center value and $\sigma_{\alpha}$ standards for $1-\sigma$ uncertainty.

Since multiple components are overlapping with each other at different visible energy regions, Markov Chain Monte Carlo (MCMC) technique is used to study the correlations among multi parameters, especially the relations between $\nu \mathrm{MM}$ and any other parameter, while simultaneously obtaining the individual posterior distribution of each component. MCMC is based on a Python package named emcee [50], which requires a likelihood function to guide Monte Carlo sampling. Therefore, we use $\mathcal{L}=\exp \left(-\frac{1}{2} \chi^{2}\right)$ to convert the $\chi^{2}$ into likelihood for MCMC.

Figure 2 presents multi-parametric scatter plots of MCMC using 10-year data taking at Jinping, showing both the correlation for each pair of parameters and their posterior distributions. The solar neutrino related parameters are shown as the ratio relative to the standard HZ flux model: $\mathrm{R}_{\phi} \odot=\phi^{\odot} / \phi_{\text {truth }}^{\odot}$. The background parameters are shown as the relative deviation of the background truth rates: $\delta \mathrm{BG}=\left(\mathrm{BG}-\mathrm{BG}_{\text {truth }}\right) / \mathrm{BG}_{\text {truth }}$. In this study, $\sin ^{2} \theta_{12}$ and $\Delta m_{21}^{2}$ are constrained with $0.54 \%$ and $0.24 \%$ respectively according to the prediction of future JUNO experiment [51]. $p p$ flux is constrained to $5 \%$ by Gallium experiments and all background parameters are free without constraints. As shown in the MCMC sampling plot, $p p$ and ${ }^{85} \mathrm{Kr}$ have the largest correlation with $\mu_{\nu}^{\text {eff }}$. Thus, good priors of $p p$ or ${ }^{85} \mathrm{Kr}$ can make corresponding improvement on the detection sensitivities of $\mu_{\nu}^{\text {eff }}$ as we expect in the analyses of previous subsection. From another point of view, the existence of $\nu \mathrm{MM}$ could also bias the measurement of $p p$ in LS experiments. In figure 2, ${ }^{210} \mathrm{Bi}$ has strong correlation with $\mathrm{CNO}$ and pep. Therefore, we could fix CNO and pep in the following study because ${ }^{210} \mathrm{Bi}$ spectrum can mimic them in the low energy region, while having little effect on the results. 


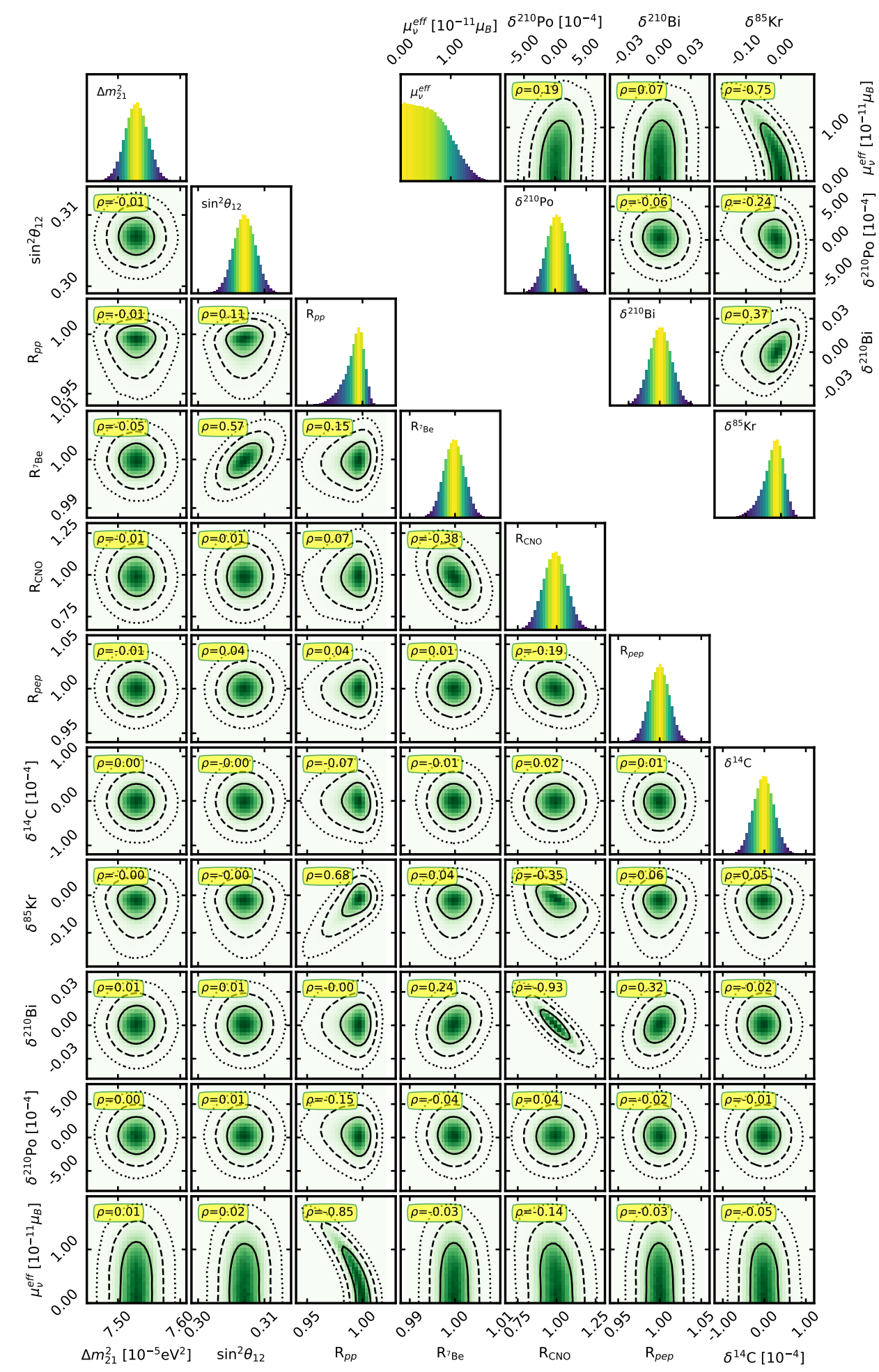

Figure 2. MCMC results on fitting parameters with 10-year data taking in Jinping. The solid, dashed and dotted lines represent $1-\sigma, 2-\sigma$ and 3- $\sigma$ contours. The diagonal plots show the posterior 1-d distribution of each parameter. Off-diagonal plots show the posterior 2-d distribution of two parameters. To fit the plot in one page, we move the bottom right triangle plots to the upper right corner of this page. 


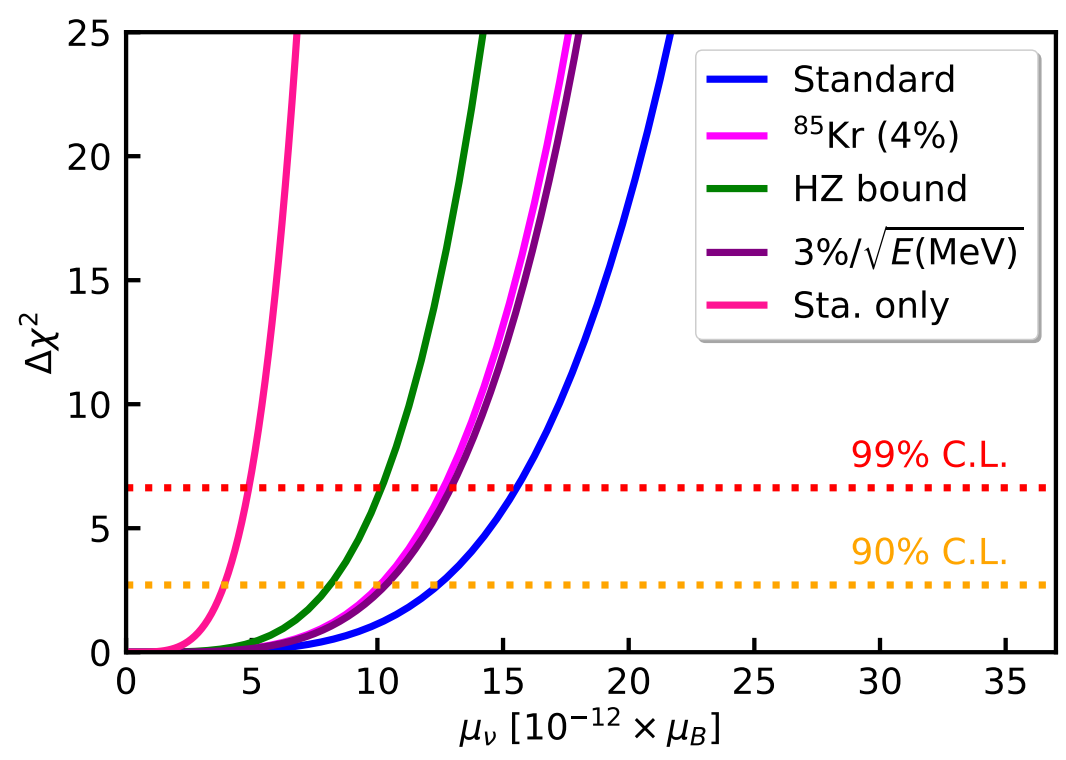

Figure 3. The sensitivity of $\nu \mathrm{MM}$ measurement at Jinping with 10-year data taking. The blue line is the standard case with only the radiochemical constraints from Gallium experiments. The fuchsia line presents the standard case with additional $4 \%$ constraint on ${ }^{85} \mathrm{Kr}$. The green line means the standard case with additional HZ model flux constraint. The purple line is the standard case with $3 \% / \sqrt{E(\mathrm{MeV})}$ energy resolution. The deep pink line is the statistics only case.

\begin{tabular}{|c|c|c|c|c|c|c|c|c|c|c|c|}
\hline Tool & $\Delta m_{21}^{2}$ & $\theta_{12}$ & $p p$ & ${ }^{7} \mathrm{Be}$ & $\mathrm{CNO}$ & $p e p$ & ${ }^{14} \mathrm{C}$ & ${ }^{85} \mathrm{Kr}$ & ${ }^{210} \mathrm{Bi}$ & ${ }^{210} \mathrm{Po}$ & $\mu_{\nu}^{\text {eff }}$ \\
\hline MCMC & $0.54 \%$ & $0.24 \%$ & $5 \%$ & free & free & free & free & free & free & free & free \\
\hline Minuit-Std & fix & fix & $5 \%$ & free & fix & fix & free & free & free & free & fix \\
\hline
\end{tabular}

Table 2. The condition of parameters in MCMC and Minuit. Minuit specifies the standard case. In MCMC, the constraints of $\Delta m_{21}^{2}$ and $\theta_{12}$ come from the future JUNO [51]. Gallium experiments constrain the $p p$ neutrino flux with about $5 \%$ in both MCMC and Minuit.

In order to study the influence of different inputs on the sensitivity to the measurement of $\nu \mathrm{MM}$, a $\chi^{2}$ based minimizer, Minuit, is used. The sensitivity of $\nu \mathrm{MM}$ is obtained for different input values of $\mu_{\nu}^{\mathrm{eff}}$, as shown in figure 3. The sensitivities to $\nu \mathrm{MM}$ from the MCMC method and $\chi^{2}$ minimizer are consistent with each other. We find that there is no difference in sensitivity to $\nu \mathrm{MM}$ between fixing solar oscillation parameters $\left(\sin ^{2} \theta_{12}\right.$ and $\Delta m_{21}^{2}$ ) and constraining them with JUNO reactor measurement. Similarly fixing or releasing $\mathrm{CNO}$ and pep also have no effect on the sensitivity. The standard case in figure 3 assumes that $\sin ^{2} \theta_{12}, \Delta m_{21}^{2}$, CNO and pep are fixed, $p p$ is bounded by Gallium experiments and other parameters are all free. Table 2 shows the fitting or sampling conditions of all parameters in Minuit and MCMC.

In figure 3 , the standard case can reach $<1.2 \times 10^{-11} \mu_{B}$ (90\% C.L.), which is already significantly better than other terrestrial experiments. Furthermore, we find that Jinping has more than $5 \sigma\left(\begin{array}{ll}3 \sigma & \sigma\end{array}\right)$ to confirm or exclude the $\nu \mathrm{MM}$ hypothesis about the recent excess in XENON1T in 10 years (4 years) for the standard case as shown in figure 3. 


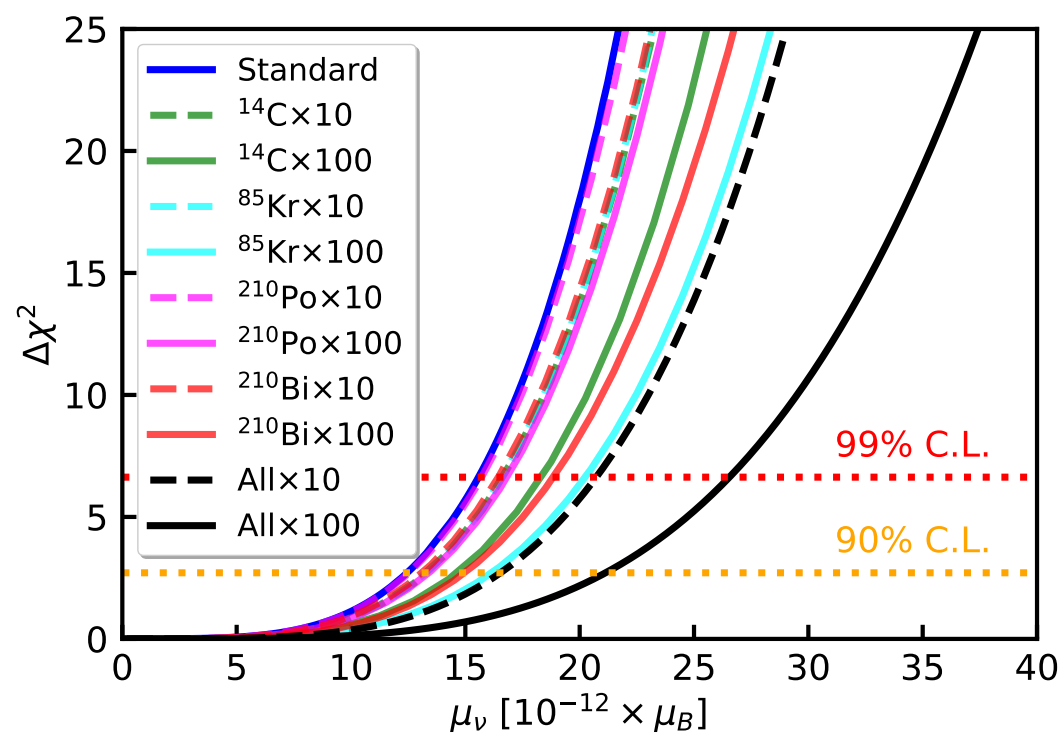

Figure 4. The sensitivity to $\nu \mathrm{MM}$ at Jinping with 10 -year data taking. The blue line is the standard case with only the radiochemical constraints from Gallium experiments. The green, cyan, fuchsia and red lines represent the sensitivity to detect $\nu \mathrm{MM}$ with increased abundance of ${ }^{14} \mathrm{C}$, ${ }^{85} \mathrm{Kr},{ }^{210} \mathrm{Po}$ and ${ }^{210} \mathrm{Bi}$ background, respectively. And the black line is for all above background. The solid (dotted) line refers a 100 (10) fold increase in the background.

Without considering systematic uncertainties, the statistical-only case shows that $\nu \mathrm{MM}$ is $<3.9 \times 10^{-12} \mu_{B}$ at $90 \%$ C.L. assuming the background level as shown in table 1. By comparing this with the standard case, we know systematic uncertainties can significantly affect the sensitivity to $\nu \mathrm{MM}$. Additional $4 \%{ }^{85} \mathrm{Kr}$ measurement can further boost the upper limit to $1 \times 10^{-11} \mu_{B}(90 \%$ C.L.) as well as $3 \% / \sqrt{E(\mathrm{MeV})}$, high light yield LS. As shown in figure 2 and $3, p p$ neutrino flux is the dominant systematic uncertainty affecting the final sensitivity. Additional $\mathrm{HZ}$ flux bound, particularly $p p$ and ${ }^{7} \mathrm{Be}$ fluxes, can improve the sensitivity on $\nu \mathrm{MM}$ down to $<0.8 \times 10^{-11} \mu_{B}$ (90\% C.L.).

In order to evaluate the effect of different background abundances on sensitivity to $\nu \mathrm{MM}$, we calculate the sensitivity to $\nu \mathrm{MM}$ with different individual reductions of ${ }^{14} \mathrm{C}$, ${ }^{85} \mathrm{Kr},{ }^{210} \mathrm{Po}$ and ${ }^{210} \mathrm{Bi}$ with simple background reductions. We find that the reduction of ${ }^{14} \mathrm{C}$ and ${ }^{85} \mathrm{Kr}$ could significantly improve the sensitivity to $\nu \mathrm{MM}$. Furthermore, we investigate how much reduction of background abundance is needed to match the $\nu \mathrm{MM}$ detection sensitivity of astrophysical observations. It would take more than 10,000-fold reduction on ${ }^{14} \mathrm{C}$ background to reach $<1.0 \times 10^{-12} \mu_{B}$ (90\% C.L.). A more than 1000 -fold reduction in ${ }^{85} \mathrm{Kr}$ background could at most reach $<6.0 \times 10^{-12} \mu_{B}$ (90\% C.L.). Any further reduction in ${ }^{85} \mathrm{Kr}$ would not help. Therefore, in order to reach the astrophysical limits, we have to heavily reduce ${ }^{14} \mathrm{C}$ and ${ }^{85} \mathrm{Kr}$ backgrounds, which is impossible with the current technology. However, any reduction of ${ }^{210} \mathrm{Po}$ and ${ }^{210} \mathrm{Bi}$ could hardly improve the sensitivity.

On the other hand, we also study the variation of $\nu \mathrm{MM}$ detection sensitivity if the abundance of backgrounds increases by a factor of 10 or 100 . As shown in figure 4, we find that even if each background is increased by 100 times individually, the detection sensitivity 


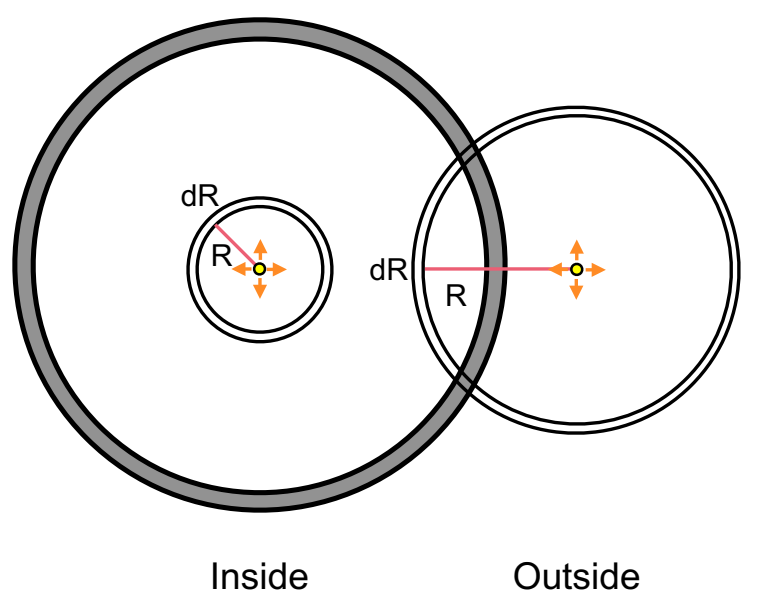

Figure 5. The cartoon sketch about the source positions. The sizes of them are not proportional to the actual sizes.

to $\nu \mathrm{MM}$ can still reach at least $<1.6 \times 10^{-11} \mu_{B}$ (90\% C.L.). Moreover, if the abundance of all those four backgrounds deteriorate to 10 times that of Borexino background level shown in table 1 , it still has $3 \sigma$ sensitivity to validate the $\nu$ MM hypothesis in XENON1T. However, the sensitivity to $\nu \mathrm{MM}$ would reduce to $<2.1 \times 10^{-11} \mu_{B}$ (90\% C.L.) with a 100 -fold increase in all background with respect to Borexino. Therefore, it is advisable to control the background abundance of Jinping within 10 times that of Borexino.

\section{$3 \quad \nu M M$ with artificial neutrino source}

As pointed out by previous study [39, 40], $\nu \mathrm{MM}$ can also be probed with artificial neutrino or antineutrino sources. The great benefit of such kind measurement is that it can largely reduce the experimental time to achieve the similar sensitivity as solar neutrinos. The largest challenging for such kind of experiment is the production, transportation and shielding for those strong radioactive sources. In the current paper, we focus on the capability of such experiment instead of worrying about the technique details.

\section{$3.1 \quad{ }^{51} \mathrm{Cr}$ neutrino signals}

According to the BEST experiment [38], we assume a $3 \mathrm{MCi}{ }^{51} \mathrm{Cr}$ source [40] can be placed at two possible locations: outside with 1 meter away from the edge of the fiducial volume or inside at the center of detector. Figure 5 shows the cartoon sketch of the proposed source positions. The decay of ${ }^{51} \mathrm{Cr}$ is ${ }^{51} \mathrm{Cr}+e^{-} \longrightarrow{ }^{51} \mathrm{~V}+\nu_{e}$, with a 27.7-day half-life. The monoenergetic neutrino energies are $752 \mathrm{keV}(9 \%), 747 \mathrm{keV}(81 \%), 432 \mathrm{keV}(1 \%)$ and $427 \mathrm{keV}(9 \%)$ respectively.

We calculate the energy spectra of signal, background and event vertex distribution as function of $R$, the distance from event vertex position to the artificial source, for both cases based. Detail of this calculation is shown in the appendix A. We obtain the effective 

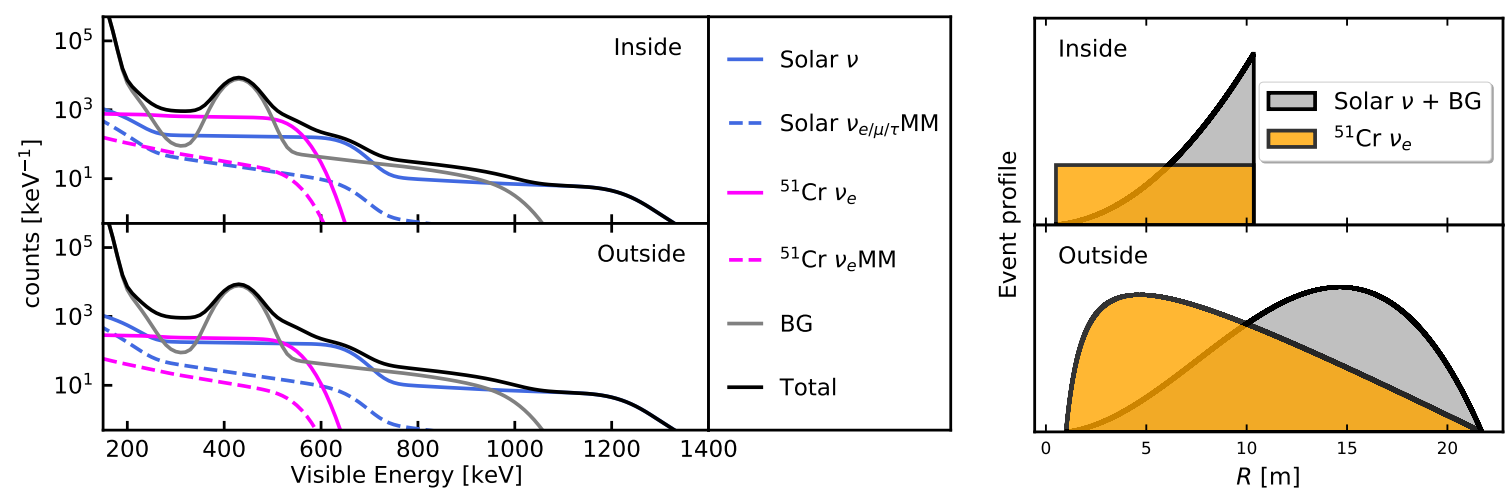

Figure 6. Left: the event number of each component by 55-day data taking with the assumption $\left(\mu_{\nu_{e}}, \mu_{\nu_{\mu}}, \mu_{\nu_{\tau}}\right)=(3.9,5.8,5.8)\left[\times 10^{-11} \mu_{B}\right]$, the individual 90\% C.L. upper limits at Borexino [25]. $4.5 \% / \sqrt{E(\mathrm{MeV})}$ energy resolution has been considered. Right: the normalized event profile with respect to $R$ from the source. The position resolution of reconstruction is not considered.

neutrino flux relative to the solar neutrinos with 55 -day ${ }^{51} \mathrm{Cr}$ source for both cases as

$$
\phi_{\text {eff }}=\left\{\begin{array}{lll}
1.27 \times 10^{10} & {\left[\mathrm{~cm}^{-2} \mathrm{~s}^{-1}\right]} & (\text { Inside }) \\
4.79 \times 10^{9} & {\left[\mathrm{~cm}^{-2} \mathrm{~s}^{-1}\right]} & (\text { Outside })
\end{array} .\right.
$$

The distributions of energy and vertex position for signal and background are shown in the left plot of figure 6 for both locations. Because of mixing, solar neutrinos contain the $\nu \mathrm{MM}$ contribution from $\nu_{e}, \nu_{\mu}$ and $\nu_{\tau}$. However, the neutrino source ${ }^{51} \mathrm{Cr}$ can only contribute the $\nu_{e} \mathrm{MM}$. Therefore, the signal of ${ }^{51} \mathrm{Cr}$ can significantly break the structure of $\nu_{e}, \nu_{\mu}$ and $\nu_{\tau}$ magnetic moment in solar neutrinos, which was also presented previously in $[25,52]$, when combined with solar neutrino signal. In this case, the probing of $\nu_{e} \mathrm{MM}$ will be more sensitive than the other two neutrino flavors. The right plot in figure 6 presents the event profile in the different slices as a function of $R$, assuming ideal position reconstruction. The position information can be used to separate the $\nu_{e}$ signal from ${ }^{51} \mathrm{Cr}$ and the solar neutrinos or background, which are uniformly distributed inside the detector.

In the left plot of figure $6,{ }^{51} \mathrm{Cr}$ shows a prominent "shoulder" structure between 500 and $600 \mathrm{keV}$, with which the flux of ${ }^{51} \mathrm{Cr}$ neutrino source could be precisely counted. Moreover, the amount of $\nu_{e} \mathrm{MM}$ shown as dashed line in fuchsia color from ${ }^{51} \mathrm{Cr}$ source is almost equal to the sum of $\nu_{e} \mathrm{MM}, \nu_{\mu} \mathrm{MM}$ and $\nu_{\tau} \mathrm{MM}$ shown as dashed line in blue color from solar neutrinos for both cases. That is to say, the sensitivity to $\nu_{e} \mathrm{MM}$ should be better than other neutrino flavor magnetic moment. The ROI of $\nu \mathrm{MM}$ with ${ }^{51} \mathrm{Cr}$ source is the same as the solar neutrino case in figure 1.

\subsection{Sensitivity}

We need to build a similar $\chi^{2}$ function as eq. (2.5) to study $\mu_{\nu_{e}}, \mu_{\nu_{\mu}}, \mu_{\nu_{\tau}}$ respectively. Given the energy spectrum and event vertex difference between signal and backgrounds, $T_{e}-R$ (visible energy and event vertex) two dimensional fitting is much better than the sensitivity from $T_{E}$ (visible energy only) one-dimensional fitting. Therefore, a two-dimensional $\chi^{2}$ 


\begin{tabular}{|c|c|c|c|c|c|c|c|c|c|c|c|c|}
\hline Tool & $\Delta m_{21}^{2}$ & $\theta_{12}$ & ${ }^{51} \mathrm{Cr}$ & $p p$ & ${ }^{7} \mathrm{Be}$ & $\mathrm{CNO}$ & $p e p$ & ${ }^{14} \mathrm{C}$ & ${ }^{85} \mathrm{Kr}$ & ${ }^{210} \mathrm{Bi}$ & ${ }^{210} \mathrm{Po}$ & $\mu_{\nu_{e, \mu, \tau}}$ \\
\hline Minuit & fix & fix & $1 \%$ & $5 \%$ & free & fix & fix & free & free & free & free & fix \\
\hline
\end{tabular}

Table 3. The status of parameters in Minuit. The constraint of ${ }^{51} \mathrm{Cr}$ comes from the independent measurements by calorimeter technique in [35, 36, 38]. Gallium experiments constrain the flux of $p p$ neutrinos with about $5 \%$.

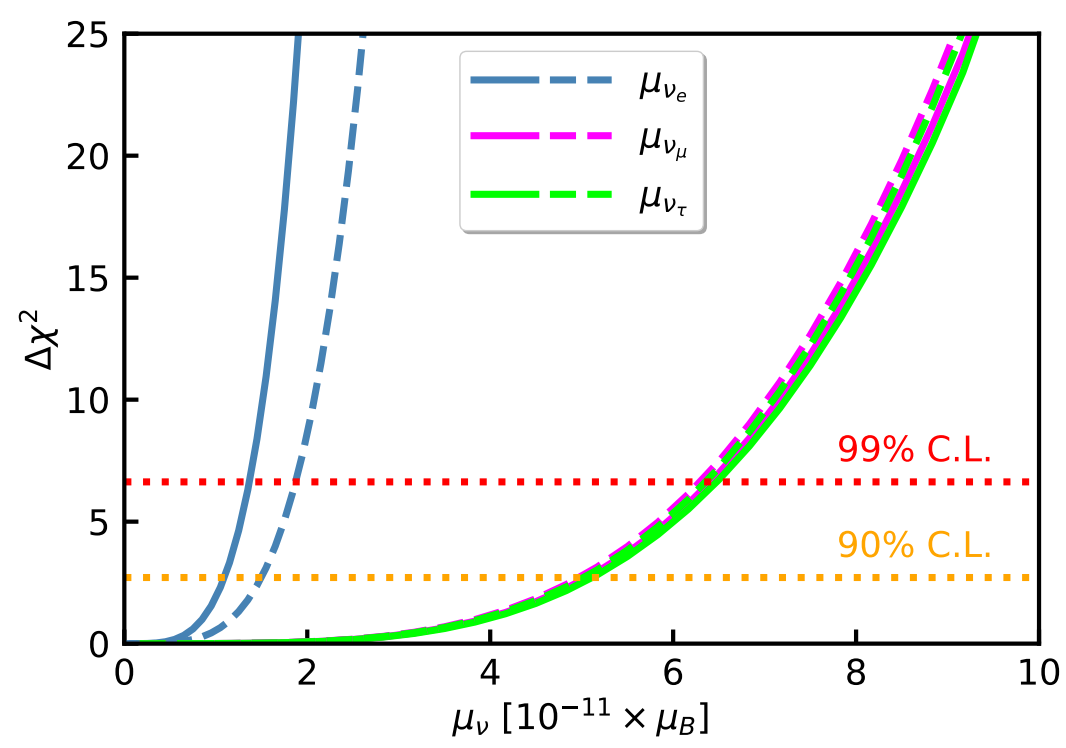

Figure 7. The sensitivity with each $\nu \mathrm{MM}$ components using 55-day data taking. The solid (dashed) lines represent the inside (outside) ${ }^{51} \mathrm{Cr}$ case.

function is used in the following study as

$$
\chi^{2}=\sum_{i}^{T_{e}} \sum_{j}^{R} \frac{\left(N_{\mathrm{pre}}^{i, j}-N_{\mathrm{obs}}^{i, j}\right)^{2}}{N_{\mathrm{obs}}^{i, j}}+\sum_{\alpha}\left(\frac{\delta_{\alpha}}{\sigma_{\alpha}}\right)^{2}
$$

where $i$ is the $i^{\text {th }} T_{e}$ bin from 150 to $1500 \mathrm{keV}$ and $j$ is the $j^{\text {th }}$ reconstructed $R$ bin. Compared with section 2, the fitter has an extra fit parameter due to the neutrino flux uncertainty from ${ }^{51} \mathrm{Cr}$. In the following analysis, we assume the uncertainty of neutrino flux from ${ }^{51} \mathrm{Cr}$ is $1 \%[35,36,38]$. Other parameters are consistent with the standard case in section 2 as shown in table 3.

Figure 7 presents the sensitivities of $\nu \mathrm{MM}$ for different neutrino flavors. The sensitivities of $\nu \mathrm{MM}$ for $\nu_{\mu}$ and $\nu_{\tau}$ are from the collected solar neutrinos during 55 days of data taking. Clearly, $\mu_{\nu_{e}}$ is the most sensitive one among all neutrino flavors due to the presence of the strong $\nu_{e}$ source. Since there is more $\nu_{e}$ signal statistics in the inside-detector case than the outside-detector case, the best sensitivity to $\mu_{\nu_{e}}$ can be achieved with the source deployed in the center of detector. On the other hand, the stronger $\nu_{e}$ neutrino flux, the weaker sensitivity to $\mu_{\nu_{\tau}}$ and $\mu_{\nu_{\tau}}$, because $\nu_{e}$ is also a background for $\mu_{\nu_{\tau}}$ and $\mu_{\nu_{\tau}}$. With 55-day data taking, we obtain the results with $90 \%$ C.L. upper limits shown in table 4. 


\begin{tabular}{|c|c|c|c|}
\hline $90 \%$ C.L. $/\left[\times 10^{-11} \mu_{B}\right]$ & $\mu_{\nu_{e}}$ & $\mu_{\nu_{\mu}}$ & $\mu_{\nu_{\tau}}$ \\
\hline Inside ${ }^{51} \mathrm{Cr}$ & 1.1 & 5.1 & 5.1 \\
\hline Outside ${ }^{51} \mathrm{Cr}$ & 1.5 & 5.0 & 5.0 \\
\hline
\end{tabular}

Table 4. $90 \%$ C.L. upper limits of $\nu \mathrm{MM}$ with 55-day data taking.
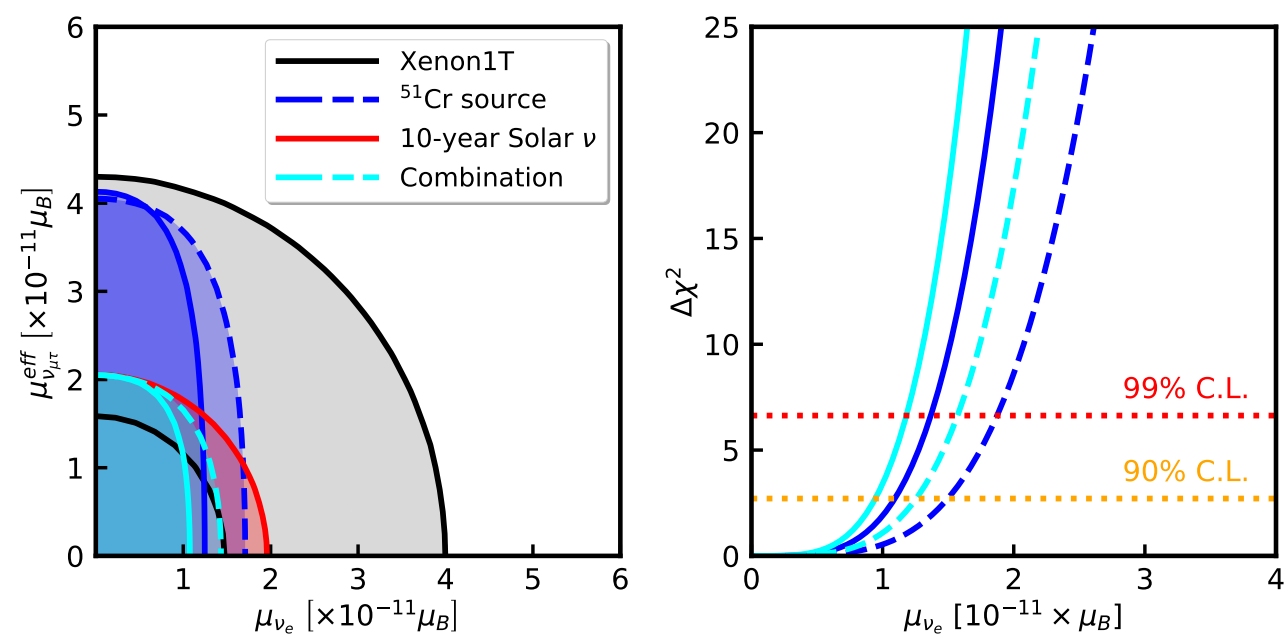

Figure 8. The left plot shows $90 \%$ C.L. upper limits on $\mu_{\nu_{e}}$ and $\mu_{\nu_{\mu \tau}}^{\text {eff }}$ for different cases. The right one is the sensitivity to $\mu_{\nu_{e}}$ after marginalization with other parameters. The gray band is the corresponding area based on 90\% C.L. in XENON1T. The solid (dashed) lines represent the inside (outside) ${ }^{51} \mathrm{Cr}$ case. The combination stands for combing the data of 10 -year solar neutrinos with 55-day ${ }^{51} \mathrm{Cr}$ source.

\subsection{Combined analyses with 55-day artificial neutrino source and 10-year solar neutrinos}

10-year data taking of solar neutrinos could give a sensitive search for $\mu_{\nu}^{\text {eff }}$, which is a combination of $\mu_{\nu_{e}}, \mu_{\nu_{\mu}}$ and $\mu_{\nu_{\tau}}$. 55 -day data taking of ${ }^{51} \mathrm{Cr}$ is more sensitive to $\mu_{\nu_{e}}$. To reach a better sensitivity, we combine the measurement of 10-year solar neutrinos and 55 -day ${ }^{51} \mathrm{Cr}$ source neutrinos. In this study, we define $\mu_{\nu_{\mu \tau}}^{\text {eff }}$, a mixing of $\mu_{\nu_{\mu}}$ and $\mu_{\nu_{\tau}}$ from solar neutrino mixing. The value of this mixed variable $\left(\mu_{\nu_{\mu \tau}}^{\mathrm{eff}}\right)^{2} \simeq 0.49 \mu_{\nu_{\mu}}^{2}+0.51 \mu_{\nu_{\tau}}^{2}$ from the appendix B.

The left plot of figure 8 shows the two dimensional 90\% C.L. contours for $\mu_{\nu_{e}}$ and $\mu_{\nu_{\mu \tau}}^{\text {eff }}$. We reproduce the XENON1T anomalous excess with $90 \%$ C.L. band of $\mu_{\nu_{e}}$ and $\mu_{\nu_{\mu \tau}}^{\text {eff }}$ for comparison. With a ${ }^{51} \mathrm{Cr}$ source, the sensitivity on $\mu_{\nu_{e}}$ improves significantly, which is even more sensitive than 10-year data taking from solar neutrinos. 55-day ${ }^{51} \mathrm{Cr}$ data could exclude most of the $\nu_{e}$ space, 10-year solar neutrinos could exclude a lot possible parameter space in both $\mu_{\nu_{e}}$ and $\mu_{\nu_{\mu \tau}}^{\text {eff }}$ plane. The right plot of figure 8 presents the sensitivity for $\mu_{\nu_{e}}$. The combination could only weakly improve $\mu_{\nu_{e}}$ to $<0.9 \times 10^{-11} \mu_{B}\left(<1.3 \times 10^{-11} \mu_{B}\right)$ (90\% C.L.) for inside case (outside case). 


\section{Conclusions}

$\nu \mathrm{MM}$ measurement plays an important role in determining the intrinsic nature of neutrinos and probing new physics in the neutrino sector. The $\nu \mathrm{MM}$ has been constrained to the $3 \times$ $10^{-11} \mu_{B}$ level at $90 \%$ C.L. by many terrestrial neutrino experiments. However, XENON1T recently reports a hint of a $\sim 2.2 \times 10^{-11} \mu_{B}$ effective neutrino magnetic moment. We have calculated the feasibility of doing $\nu \mathrm{MM}$ measurement at Jinping neutrino experiment with both natural and artificial neutrino sources.

We find the sensitivity of $\mu_{\nu}^{\text {eff }}$ can reach $<1.2 \times 10^{-11} \mu_{B}$ level at $90 \%$ C.L. with 10 -year data taking from solar neutrinos at Jinping, which can validate the $\nu$ MM hypothesis in XENON1T by more than $5 \sigma$. A $4 \%$ bound on ${ }^{85} \mathrm{Kr}$ or $3 \% / \sqrt{E(\mathrm{MeV})}$ energy resolution could improve the sensitivity to $<1.0 \times 10^{-11} \mu_{B}$ (90\% C.L.). $p p$ Neutrino flux is the dominant systematic uncertainty that affects the sensitivity to $\nu \mathrm{MM}$. The HZ model constraints on the fluxes of $p p$ and ${ }^{7} \mathrm{Be}$ could lead to $<0.8 \times 10^{-11} \mu_{B}$ (90\% C.L.). In addition, we find that the reduction of ${ }^{14} \mathrm{C}$ could significantly improve the sensitivity to $\nu \mathrm{MM}$ detection, while the reduction of ${ }^{85} \mathrm{Kr}$ only has a limited improvement. On the other hand, to be conservative, it is advisable to control the background abundance of Jinping within 10 times that of Borexino. In this case, Jinping still has $3 \sigma$ to validate the $\nu \mathrm{MM}$ hypothesis in XENON1T.

With respect to $3 \mathrm{MCi}{ }^{51} \mathrm{Cr}$ neutrino source, $\nu_{e} \mathrm{MM}$ can be measured with $<1.1 \times$ $10^{-11} \mu_{B}\left(<1.5 \times 10^{-11} \mu_{B}\right)$ at $90 \%$ C.L. for inside (outside) the detector in 55 days. We have also considered the combination of 10 -year solar neutrino and 55 -day ${ }^{51} \mathrm{Cr}$ to determine the neutrino magnetic moment induced by different neutrino flavors.

In the end, we present the current several best results of $\nu \mathrm{MM}$ and the sensitivities of both solar neutrino and artificial neutrino sources at Jinping in figure 9. The left segment of figure 9 shows $\mu_{\nu}^{\text {eff }}$ from this work compared to terrestrial experiments: Borexino [25], XENON1T [26] and PandaX-II [27], also with the astrophysical observations: cooling of globular clusters [30], white dwarfs [31] and red clump stars [53]. The right segment presents the electronic neutrino magnetic moment from this work with ${ }^{51} \mathrm{Cr}$ source compared to Gemma experiment [24]. As shown in figure 9, Jinping could validate the $\nu$ MM hypothesis as suggested by XENON1T in the future. It could reach the region of astrophysical interest by reducing the systematic uncertainties and the intrinsic background or by enriching the strength of ${ }^{51} \mathrm{Cr}$ source.

\section{Acknowledgments}

We would like to express the gratitude to Shaomin Chen and Zhe Wang for their insight and discussion for the Jinping Neutrino Experiment. Jiajie Ling acknowledges the support from National Key R\&D program of China under grant NO. 2018YFA0404013, National Natural Science Foundation of China under Grant NO. 11775315, Key Lab of Particle \& Radiation Imaging, Ministry of Education. Jiajun Liao acknowledges the support from the National Natural Science Foundation of China (Grant No. 11905299), Guangdong Basic and Applied 


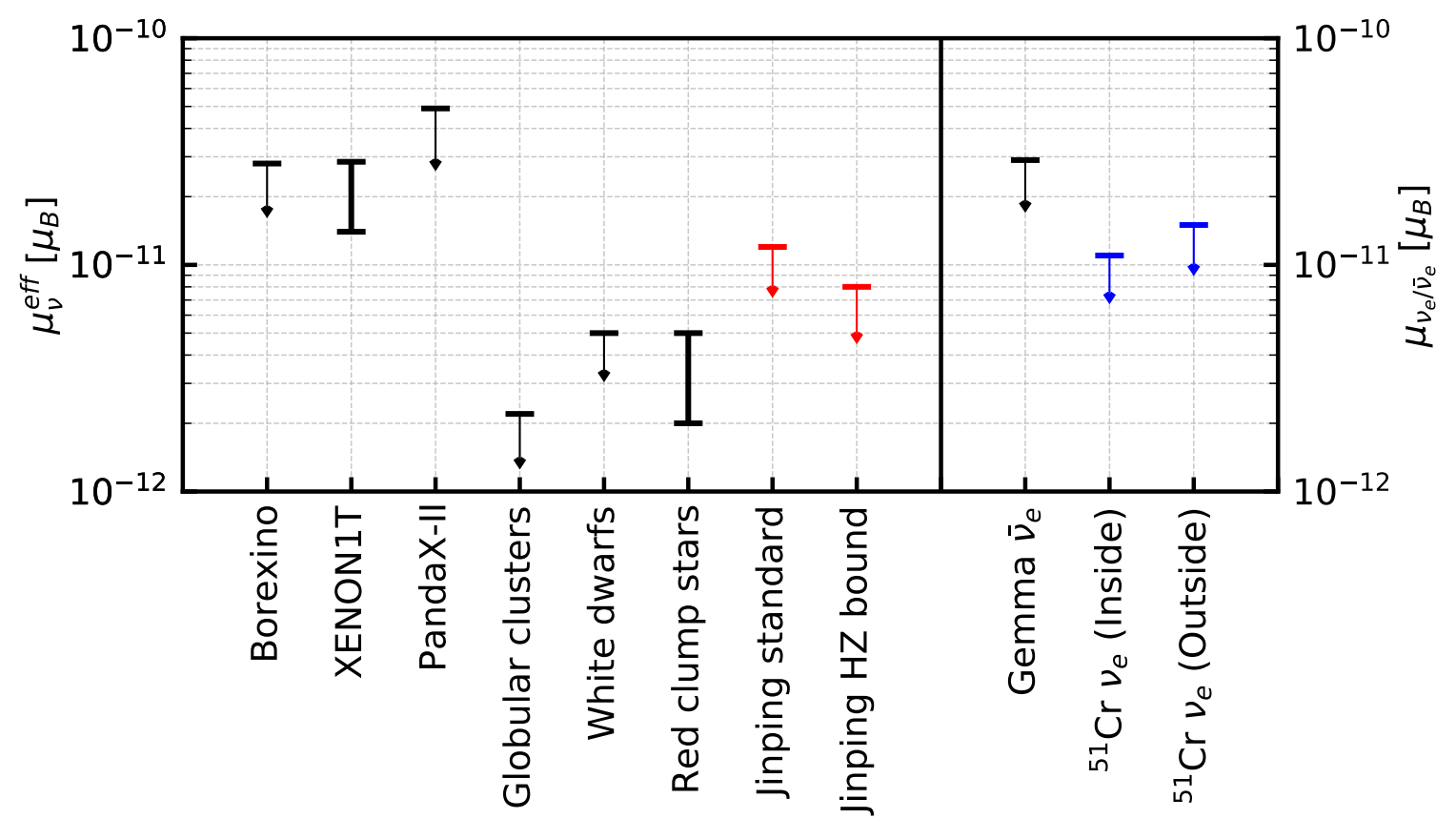

Figure 9. The current status (90\% C.L.) of the neutrino magnetic moment and the sensitivities of Jinping both with solar neutrinos and artificial source.

Basic Research Foundation (Grant No. 2020A1515011479), the Fundamental Research Funds for the Central Universities, and the Sun Yat-Sen University Science Foundation.

Note added. During preparing this paper, we notice an independent and similar study arXiv:2103.11771 is also proposed and studied by Z. Ye et al. simultaneously.

\section{A Electron scattering signal distribution and the effective neutrino flux calculation for ${ }^{51} \mathrm{Cr}$ source}

Outside source. We predict the signal event in different interfacial shell centered on the source with a radius $R$ and a thickness $\mathrm{d} R$ as

$$
N\left(R, t, T_{e}\right)=N_{e}(R) \sum_{i} \psi\left(R, t, E_{i}\right) \sum_{\alpha=e, \mu, \tau} P_{e \alpha}\left(E_{i}\right) \sigma_{\alpha}\left(E_{i}, T_{e}\right)
$$

where $N_{e}(R)$ is the total electron number density as a function of $R$ in the shell, $\psi\left(R, t, E_{i}\right)$ is the neutrino flux as a function of radius $R$, time $t$ and the $i^{\text {th }}$ neutrino branch with different monoenergetic $E_{i}$. This formula can also be used for the light sterile neutrino study such as ref. [53]. In our case, there is almost no neutrino oscillation. Therefore, it can reduce to

$$
N\left(R, t, T_{e}\right)=N_{e}(R) \sum_{i} \psi\left(R, t, E_{i}\right) \sigma_{e}\left(E_{i}, T_{e}\right),
$$

where $N_{e}(R)=S(R) \rho_{\mathrm{LS}} \rho_{e} \mathrm{~N}_{\mathrm{A}}$ with the area of the shell $S(R)$ and $\psi\left(R, t, E_{i}\right)=f\left(E_{i}\right) \phi(R, t)$ $=f\left(E_{i}\right) \frac{R_{51} \mathrm{Cr}(t)}{4 \pi R^{2}} . f\left(E_{i}\right)$ is the fraction of the $i^{\text {th }}$ branch and $R_{51} \mathrm{Cr}(t)$ is the decay rate initialized with $3 \mathrm{MCi}$. The area of the shell $S(R)$ is expressed by $S(R)=2 \pi\left(1-\frac{\left(R_{0}+x\right)^{2}+R^{2}-R_{0}^{2}}{2\left(R_{0}+x\right) R}\right)$, 
where $R_{0}(\sim 10.4 \mathrm{~m})$ is the radius of the fiducial volume sphere and $x(1 \mathrm{~m})$ is the shortest distance from the source to the edge of the fiducial volume. So far, we obtain the total event number as a function of time $t$ and $T_{e}$ with the integral by $R$ from $x$ to $x+2 R_{0}$

$$
N\left(t, T_{e}\right)=\frac{1}{2}\left[R_{0}-\frac{\left(R_{0}+x\right)^{2}-R_{0}^{2}}{2\left(R_{0}+x\right)} \ln \frac{x+2 R_{0}}{x}\right] \rho_{\mathrm{LS}} \rho_{e} \mathrm{~N}_{\mathrm{A}} R_{51 \mathrm{Cr}}(t) \sum_{i} f\left(E_{i}\right) \sigma_{e}\left(E_{i}, T_{e}\right) .
$$

If utilizing an effective ${ }^{51} \mathrm{Cr}$ decay rate $R_{51}^{\text {eff }} \mathrm{Cr}$ during $T=55$ days, it reduces to

$$
N\left(T_{e}\right)=\frac{1}{2}\left[R_{0}-\frac{\left(R_{0}+x\right)^{2}-R_{0}^{2}}{2\left(R_{0}+x\right)} \ln \frac{x+2 R_{0}}{x}\right] \rho_{\mathrm{LS}} \rho_{e} \mathrm{~N}_{\mathrm{A}} R_{51}^{\mathrm{eff}} T \sum_{i} f\left(E_{i}\right) \sigma_{e}\left(E_{i}, T_{e}\right),
$$

where $R_{51}^{\text {eff }}$ Cr $=6.005 \times 10^{16} \mathrm{~Bq}$. Moreover, an effective neutrino flux $\phi_{\text {eff with respect to }}$ the whole fiducial volume can be written as $\phi_{\text {eff }}=\frac{3}{8 \pi}\left[\frac{1}{R_{0}^{2}}-\frac{\left(R_{0}+x\right)^{2}-R_{0}^{2}}{2\left(R_{0}+x\right) R_{0}^{3}} \ln \frac{x+2 R_{0}}{x}\right] R_{51}^{\mathrm{eff}}$ ir ${ }^{\text {so }}$ as to compare with the fluxes of solar neutrinos.

Inside source. With the same calculation strategy, the signal event in different spherical shell yields

$$
N\left(R, t, T_{e}\right)=\rho_{\mathrm{LS}} \rho_{e} \mathrm{~N}_{\mathrm{A}} R_{51} \mathrm{Cr}(t) \sum_{i} f\left(E_{i}\right) \sigma_{e}\left(E_{i}, T_{e}\right),
$$

which is an uniform distribution of $R$. With an integral by $R$ and $t$, we obtain

$$
N\left(T_{e}\right)=\rho_{\mathrm{LS}} \rho_{e} \mathrm{~N}_{\mathrm{A}} R_{51}^{\mathrm{eff}}\left(R_{0}-r\right) T \sum_{i} f\left(E_{i}\right) \sigma_{e}\left(E_{i}, T_{e}\right),
$$

where $r$ is the radius $(0.5 \mathrm{~m})$ of the sphere source shielding. In addition, we obtain an effective neutrino flux $\phi_{\text {eff }}=\frac{3}{4 \pi\left(R_{0}^{2}+r R_{0}+r^{2}\right)} R_{51}^{\text {eff }}$ to compare with the solar neutrino fluxes.

\section{B An effective neutrino magnetic moment mixing $\mu_{\nu \tau}^{\text {eff }}$}

We define $\mu_{\nu_{\mu \tau}}^{\mathrm{eff}}$ as an effective mixing of $\mu_{\nu_{\mu}}$ and $\mu_{\nu_{\tau}}$. Therefore, eq. (2.2) can be modified as

$$
N_{\mathrm{pre}}=N_{e} \sum_{i} \phi_{i} \iint S_{i}^{\odot}(E)\left(P_{e e}^{i}(E) \sigma_{e}\left(E, T_{e}\right)+\left(P_{e \mu}^{i}(E)+P_{e \tau}^{i}(E)\right) \sigma_{\mu \tau}\left(E, T_{e}\right)\right) d E d T_{e},
$$

with $\sigma_{\mu \tau}=\sigma_{\mu \tau}^{\mathrm{SM}}+\sigma_{\mu \tau}^{\nu_{\mu \tau} \mathrm{MM}}$, and $\sigma_{\mu \tau}^{\nu_{\mu \tau} \mathrm{MM}}=\pi \frac{\alpha^{2}}{m_{e}^{2}}\left(\frac{\mu_{\nu \mu \tau}^{\mathrm{eff}}}{\mu_{B}}\right)^{2}\left(\frac{1}{T_{e}}-\frac{1}{E_{\nu}}\right)$. Moreover, remove the $\mathrm{SM}$ and $\nu_{e} \mathrm{MM}$ contributions from the event number, resulting in

$$
\begin{aligned}
& \sum_{i} \phi_{i} \iint \pi \frac{\alpha^{2}}{m_{e}^{2}} S_{i}^{\odot}(E)\left(P_{e \mu}^{i}(E)+P_{e \tau}^{i}(E)\right)\left(\frac{\mu_{\nu_{\mu \tau}}^{\mathrm{eff}}}{\mu_{B}}\right)^{2}\left(\frac{1}{T_{e}}-\frac{1}{E_{\nu}}\right) d E d T_{e} \\
& =\sum_{i} \phi_{i} \iint \pi \frac{\alpha^{2}}{m_{e}^{2}} S_{i}^{\odot}(E)\left(P_{e \mu}^{i}(E)\left(\frac{\mu_{\nu_{\mu}}}{\mu_{B}}\right)^{2}+P_{e \tau}^{i}(E)\left(\frac{\mu_{\nu_{\tau}}}{\mu_{B}}\right)^{2}\right)\left(\frac{1}{T_{e}}-\frac{1}{E_{\nu}}\right) d E d T_{e},
\end{aligned}
$$

where $\mu_{\nu_{\mu \tau}}^{\text {eff }}$ can be split into $\mu_{\nu_{\mu}}$ and $\mu_{\nu_{\tau}}$. In this study, ${ }^{7} \mathrm{Be}(862 \mathrm{keV})$ dominates the proportions of $\mu_{\nu_{\mu}}$ and $\mu_{\nu_{\tau}}$ in $\mu_{\nu_{\mu \tau}}^{\text {eff }}$ at ROI, the yellow band in figure 1. Therefore, eq. (B.2) can reduce to

$$
\left(P_{e \mu}^{7} \mathrm{Be}+P_{e \tau}^{7} \mathrm{Be}\right)\left(\mu_{\nu_{\mu \tau}}^{\mathrm{eff}}\right)^{2} \simeq P_{e \mu}^{7} \mathrm{Be} \mu_{\nu_{\mu}}^{2}+P_{e \tau}^{7} \mathrm{Be} \mu_{\nu_{\tau}}^{2},
$$


where $P_{e \alpha}^{7 \mathrm{Be}}$ obeys eq. (2.3). Further more, the average oscillation probability $P_{e \alpha}^{7 \mathrm{~B} e}$ approximates $P_{e \alpha}\left(r=0.06 R_{\odot}, E=862 \mathrm{keV}\right)$, the probability from the densest point of ${ }^{7} \mathrm{Be}$ production in the Sun to the Earth. Therefore, we obtain $\left(\mu_{\nu_{\mu \tau}}^{\mathrm{eff}}\right)^{2} \simeq 0.49 \mu_{\nu_{\mu}}^{2}+0.51 \mu_{\nu_{\tau}}^{2}$ with the neutrino oscillation parameters in ref. [44].

Open Access. This article is distributed under the terms of the Creative Commons Attribution License (CC-BY 4.0), which permits any use, distribution and reproduction in any medium, provided the original author(s) and source are credited.

\section{References}

[1] L.B. Okun, M.B. Voloshin and M.I. Vysotsky, Electromagnetic Properties of Neutrino and Possible Semiannual Variation Cycle of the Solar Neutrino Flux, Sov. J. Nucl. Phys. 44 (1986) 440 [INSPIRE].

[2] L.B. Okun, M.B. Voloshin and M.I. Vysotsky, Neutrino Electrodynamics and Possible Effects for Solar Neutrinos, Sov. Phys. JETP 64 (1986) 446 [INSPIRE].

[3] C.-S. Lim and W.J. Marciano, Resonant Spin-Flavor Precession of Solar and Supernova Neutrinos, Phys. Rev. D 37 (1988) 1368 [InSPIRE].

[4] E.K. Akhmedov, Resonant Amplification of Neutrino Spin Rotation in Matter and the Solar Neutrino Problem, Phys. Lett. B 213 (1988) 64 [INSPIRE].

[5] H.O. Back et al., Study of the neutrino electromagnetic properties with prototype of Borexino detector, Phys. Lett. B 563 (2003) 35 [InSPIRE].

[6] W. Grimus, M. Maltoni, T. Schwetz, M.A. Tortola and J.W.F. Valle, Constraining Majorana neutrino electromagnetic properties from the LMA-MSW solution of the solar neutrino problem, Nucl. Phys. B 648 (2003) 376 [hep-ph/0208132] [INSPIRE].

[7] C. Giunti and A. Studenikin, Neutrino electromagnetic interactions: a window to new physics, Rev. Mod. Phys. 87 (2015) 531 [arXiv: 1403.6344] [InSPIRE].

[8] K. Fujikawa and R. Shrock, The Magnetic Moment of a Massive Neutrino and Neutrino Spin Rotation, Phys. Rev. Lett. 45 (1980) 963 [InSPIRE].

[9] J. Schechter and J.W.F. Valle, Majorana Neutrinos and Magnetic Fields, Phys. Rev. D 24 (1981) 1883 [Erratum ibid. 25 (1982) 283] [INSPIRE].

[10] B. Kayser, Majorana Neutrinos and their Electromagnetic Properties, Phys. Rev. D 26 (1982) 1662 [INSPIRE].

[11] J.F. Nieves, Electromagnetic Properties of Majorana Neutrinos, Phys. Rev. D 26 (1982) 3152 [INSPIRE].

[12] P.B. Pal and L. Wolfenstein, Radiative Decays of Massive Neutrinos, Phys. Rev. D 25 (1982) 766 [INSPIRE].

[13] R.E. Shrock, Electromagnetic Properties and Decays of Dirac and Majorana Neutrinos in a General Class of Gauge Theories, Nucl. Phys. B 206 (1982) 359 [InSPIRE].

[14] KATRIN collaboration, Improved Upper Limit on the Neutrino Mass from a Direct Kinematic Method by KATRIN, Phys. Rev. Lett. 123 (2019) 221802 [arXiv:1909.06048] [INSPIRE]. 
[15] M. Fukugita and T. Yanagida, A Particle Physics Model for Voloshin-Vysotskii-Okun Solution to the Solar Neutrino Problem, Phys. Rev. Lett. 58 (1987) 1807 [InSPIRE].

[16] S. Pakvasa and J.W.F. Valle, Neutrino properties before and after KamLAND, Proc. Indian Natl. Sci. Acad. A $\mathbf{7 0}$ (2004) 189 [hep-ph/0301061] [InSPIRE].

[17] M. Gorchtein, N.F. Bell, M.J. Ramsey-Musolf, P. Vogel and P. Wang, Model Independent Naturalness Bounds on Magnetic Moments of Majorana Neutrinos, AIP Conf. Proc. 903 (2007) 287 [hep-ph/0610388] [INSPIRE].

[18] N.F. Bell, M. Gorchtein, M.J. Ramsey-Musolf, P. Vogel and P. Wang, Model independent bounds on magnetic moments of Majorana neutrinos, Phys. Lett. B 642 (2006) 377 [hep-ph/0606248] [INSPIRE].

[19] N.F. Bell, V. Cirigliano, M.J. Ramsey-Musolf, P. Vogel and M.B. Wise, How magnetic is the Dirac neutrino?, Phys. Rev. Lett. 95 (2005) 151802 [hep-ph/0504134] [INSPIRE].

[20] N.F. Bell, V. Cirigliano, M.J. Ramsey-Musolf, P. Vogel and M.B. Wise, Magnetic moments of Dirac neutrinos, AIP Conf. Proc. 842 (2006) 874 [hep-ph/0601005] [InSPIRE].

[21] B. Kayser, Neutrino properties, in Proceedings of "The Neutrino 08", Christchurch, New Zealand, 25-31 May 2008.

[22] C. Giunti and A. Studenikin, Neutrino electromagnetic properties, Phys. Atom. Nucl. 72 (2009) 2089 [arXiv: 0812.3646] [INSPIRE].

[23] SuPER-KamioKande collaboration, Limits on the neutrino magnetic moment using 1496 days of Super-Kamiokande-I solar neutrino data, Phys. Rev. Lett. 93 (2004) 021802 [hep-ex/0402015] [INSPIRE].

[24] A.G. Beda et al., Gemma experiment: The results of neutrino magnetic moment search, Phys. Part. Nucl. Lett. 10 (2013) 139.

[25] Borexino collaboration, Limiting neutrino magnetic moments with Borexino Phase-II solar neutrino data, Phys. Rev. D 96 (2017) 091103 [arXiv:1707.09355] [INSPIRE].

[26] XENON collaboration, Excess electronic recoil events in XENON1T, Phys. Rev. D 102 (2020) 072004 [arXiv: 2006. 09721] [INSPIRE].

[27] PANDAX-II collaboration, A search for solar axions and anomalous neutrino magnetic moment with the complete PandaX-II data, arXiv:2008.06485 [INSPIRE].

[28] G.G. Raffelt, Core Mass at the Helium Flash From Observations and a New Bound on Neutrino Electromagnetic Properties, Astrophys. J. 365 (1990) 559 [INSPIRE].

[29] S. Arceo-Díaz, K.P. Schröder, K. Zuber and D. Jack, Constraint on the magnetic dipole moment of neutrinos by the tip-RGB luminosity in $\omega$-Centauri, Astropart. Phys. 70 (2015) 1 [INSPIRE].

[30] S.A. Díaz, K.-P. Schröder, K. Zuber, D. Jack and E.E.B. Barrios, Constraint on the axion-electron coupling constant and the neutrino magnetic dipole moment by using the tip-RGB luminosity of fifty globular clusters, arXiv:1910.10568 [INSPIRE].

[31] A.H. Córsico, L.G. Althaus, M.M. Miller Bertolami, S.O. Kepler and E. García-Berro, Constraining the neutrino magnetic dipole moment from white dwarf pulsations, JCAP 08 (2014) 054 [arXiv: 1406.6034] [INSPIRE].

[32] JinPING collaboration, Physics prospects of the Jinping neutrino experiment, Chin. Phys. C 41 (2017) 023002 [arXiv: 1602.01733] [INSPIRE]. 
[33] J.N. Abdurashitov et al., Measurement of the response of a Ga solar neutrino experiment to neutrinos from an Ar-37 source, Phys. Rev. C 73 (2006) 045805 [nucl-ex/0512041] [INSPIRE].

[34] GALLEX collaboration, Final results of the ${ }^{51} \mathrm{Cr}$ neutrino source experiments in GALLEX, Phys. Lett. B 420 (1998) 114 [inSPIRE].

[35] SAGE collaboration, Measurement of the response of the Russian-American gallium experiment to neutrinos from a ${ }^{51} \mathrm{Cr}$ source, Phys. Rev. C 59 (1999) 2246 [hep-ph/9803418] [INSPIRE].

[36] M. Cribier et al., Production of a 62 $\mathrm{PBq}{ }^{51} \mathrm{Cr}$ low-energy neutrino source for GALLEX, Nucl. Instrum. Meth. A 378 (1996) 233 [INSPIRE].

[37] V. Gavrin, SAGE and BEST, talk at The XXIX International Conference on Neutrino Physics, 23 June 2020.

[38] V.N. Gavrin, T.V. Ibragimova, J.P. Kozlova, V.A. Tarasov, E.P. Veretenkin and A.I. Zvir, Measurement of neutrino source activity in the experiment BEST by calorimetric method, 2021 JINST 16 P04012 [arXiv: 2105.05120] [INSPIRE].

[39] P. Coloma, P. Huber and J.M. Link, Combining dark matter detectors and electron-capture sources to hunt for new physics in the neutrino sector, JHEP 11 (2014) 042 [arXiv: 1406.4914] [INSPIRE].

[40] P. Coloma, P. Huber and J.M. Link, Telling Solar Neutrinos from Solar Axions When You Can't Shut Off the Sun, arXiv:2006.15767 [INSPIRE].

[41] Z. Wang, private communication.

[42] N. Vinyoles et al., A new Generation of Standard Solar Models, Astrophys. J. 835 (2017) 202 [arXiv: 1611.09867] [INSPIRE].

[43] J.N. Bahcall, A.M. Serenelli and S. Basu, 10,000 standard solar models: a Monte Carlo simulation, Astrophys. J. Suppl. 165 (2006) 400 [astro-ph/0511337] [INSPIRE].

[44] Particle Data Group collaboration, Review of Particle Physics, PTEP 2020 (2020) 083C01 [INSPIRE].

[45] SAGE collaboration, Measurement of the solar neutrino capture rate with gallium metal. III: Results for the 2002-2007 data-taking period, Phys. Rev. C 80 (2009) 015807 [arXiv:0901.2200] [INSPIRE].

[46] Borexino collaboration, First Simultaneous Precision Spectroscopy of pp, ${ }^{7}$ Be, and pep Solar Neutrinos with Borexino Phase-II, Phys. Rev. D 100 (2019) 082004 [arXiv: 1707.09279] [INSPIRE].

[47] Borexino collaboration, The Monte Carlo simulation of the Borexino detector, J. Phys. Conf. Ser. 1342 (2020) 012035 [INSPIRE].

[48] BorexiNo collaboration, Neutrinos from the primary proton-proton fusion process in the Sun, Nature 512 (2014) 383 [INSPIRE].

[49] V.V. Kuzminov and N.Y. Osetrova, Precise measurement of ${ }^{14} \mathrm{C}$ beta spectrum by using a wall-less proportional counter, Phys. Atom. Nucl. 63 (2000) 1292 [INSPIRE].

[50] D. Foreman-Mackey, D.W. Hogg, D. Lang and J. Goodman, emcee: The MCMC Hammer, Publ. Astron. Soc. Pac. 125 (2013) 306 [arXiv:1202.3665] [INSPIRE]. 
[51] JUNO collaboration, Neutrino Physics with JUNO, J. Phys. G 43 (2016) 030401 [arXiv : 1507.05613] [INSPIRE].

[52] A.N. Khan, $\sin ^{2} \theta_{W}$ Estimate and Neutrino Electromagnetic Properties from Low-Energy Solar Data, J. Phys. G 46 (2019) 035005 [arXiv: 1709. 02930] [inSPIRE].

[53] M.V. Smirnov, Z.J. Hu, J.J. Ling, Y.N. Novikov, Z. Wang and G. Yang, Sterile neutrino oscillometry with Jinping, Eur. Phys. J. C 80 (2020) 609 [arXiv: 2002.05246] [InSPIRE]. 